\title{
Feature
}

\section{Video Views and Reviews: Creating a Thread with Respect to the Invasion of Animal Viruses}

\section{Christopher Watters}

\author{
Department of Biology, Middlebury College, Bicentennial Way, Middlebury, VT 05753
}

In previous columns I have mentioned using conceptually related research articles and videos as the basis for seminar or journal club discussions. Here I review material that might be used for such purposes that deals with the infection pathways taken by three different animal viruses. The comparative study would be useful for discussions involving intermediate and advanced undergraduates, but given a bit of background, introductory students could readily appreciate the first two movies. Before discussing the videos, I first describe the "thread" I followed in selecting these research records, and, as always, I appreciate hearing your reactions to these reviews and your suggestions of other peer-reviewed videos for possible review as educational material.

I began preparing this article by scanning the Annotated Video Collection from The Journal of Cell Biology Web site (www.jcb.org), which contains brief descriptions of research videos organized by cellular categories. For their timeliness and broad interest, I selected videos from the "Pathogenesis" section that characterized the intracellular behavior of the human immunodeficient virus (HIV) in host cells (McDonald et al., 2002). I then explored more recently published articles that cited that paper, which I found appended to the end of the paper. The list was prepared by HighWire Press (the service that helps The Journal of Cell Biology and other journals publish online) with online links to each paper. I used one of these links to reach a study in the Proceedings of the National Academy of Sciences, USA (PNAS) by Lakadamyali et al. (2003), which contained videos depicting the endosomal pathway followed by the influenza virus. Making use of the HighWire citation list appended to the PNAS article, I found an excellent review of viral entry mechanisms (Smith and Helenius, 2004), which although lacking video records contained an extensive bibliography. A search of the Smith and Helenius bibliography uncovered an earlier article from the Helenius lab containing numerous video records that document the involvement of actin and dynamin on entry of the SV-40 virus into host cells (Pelkmans et al., 2002). These three articles present a contemporary view of the infection pathways taken by three different animal viruses, and my online search producing this thread took less than an hour. The thread could be expanded easily to encompass a broader

DOI: $10.1187 /$ cbe.04-07-0048

E-mail address: watters@middlebury.edu. comparative study of virus invasion mechanisms, and it could be updated routinely in future years by searching for research articles that cite the review by Smith and Helenius (2004). A similar bibliographic strategy could be used for establishing other discussion threads.

\section{VISUALIZING HIV IN LIVING CELLS}

$\mathrm{HIV}$, like other retroviruses, infects a host cell through fusion of its viral membrane (VM) with the plasma membrane (PM) of the host cell in a process resembling "exocytosis" in reverse (see Alberts et al., 2002). Following infection, the HIV core of single-stranded RNA and protein is modified and the RNA genome is replicated by reverse transcriptase into DNA; and that DNA copy is inserted into the host cell genome as a "provirus." Subsequent HIV replication thus entails a process similar to eukaryotic transcription, with the provirus as template. Host cell pathways are co-opted by HIV for the synthesis and insertion of PM proteins for VM proteins and for the synthesis of cytoplasmic proteins for viral core proteins (see Goldsby et al., 2003). Targeting of the viral components coming into and leaving the host cell is a complex phenomenon, and although much is known of a few individual steps in the pathway, the overall process, including infection and provirus formation, has not been well characterized.

To investigate the postinfection pathway followed by the virus, McDonald et al. (2002) created HIV particles containing green fluorescent protein fused to the core accessory protein $\mathrm{Vpr}$ (GFP-Vpr) and a fluorescent red lipophilic marker (DiD) to stain the VM. Human fibroblasts (Hos) were artificially infected at high titer, and the behavior of the tagged viruses was followed by fluorescence microscopy. Green particles lacking red DiD-positive VM were assumed to have successfully "infected" host cells following VM-PM fusion, whereas red particles retaining their DiD-positive VM were assumed to have been unsuccessful invaders (although presumably still attached to the host cell PM). The cells were also stained with rhodamine-labeled tubulin, which caused microtubules to appear blue. Figure 1 illustrates three stills taken from the authors' videos $2 \mathrm{~A}$ and $2 \mathrm{~B}$, showing the behavior of green core particles moving independently of (yellow arrow) or in association with (white arrow) the blue microtubular cytoskeleton.

Examining Figures $1 \mathrm{~B}$ and $\mathrm{C}$ (or the larger suite of images in the authors' figure 4B), most students will immediately appreciate both the directional movement of the HIV particle 


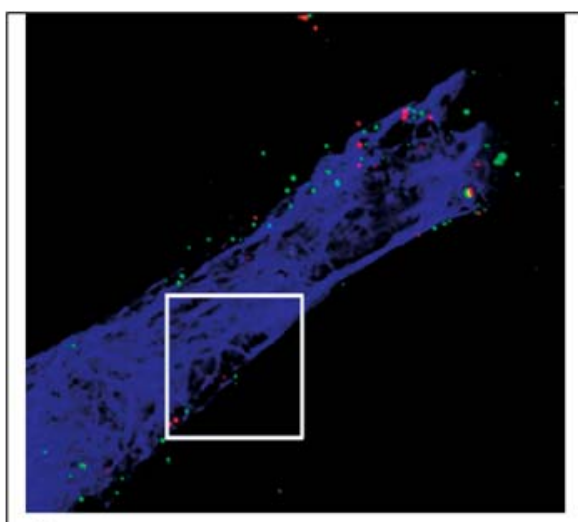

A

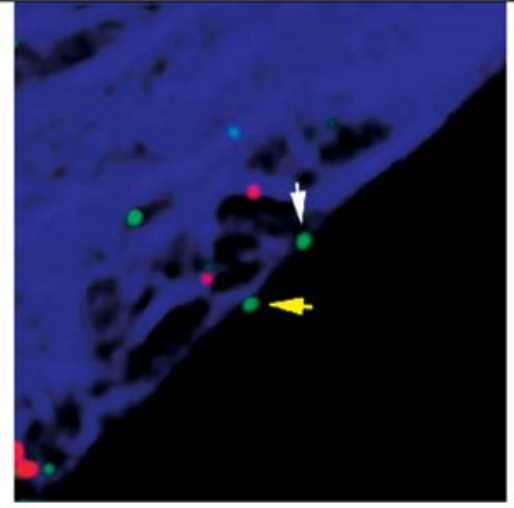

B

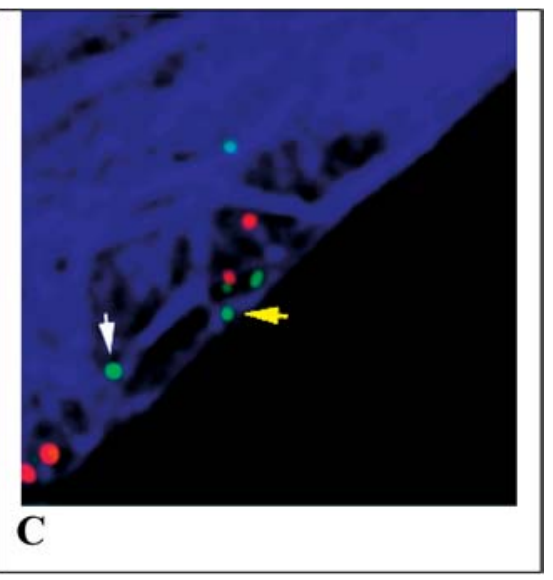

Figure 1. Fluorescence microscope images of Hos/CD4 cells containing rhodamine-labeled tubulin (blue), membrane-denuded GFP-Vprlabeled HIV (green) and membrane-encapsulated DiD-labeled HIV (red). The arrows show the locations of viral particles thought to be moving independently of (yellow) or in association with (white) microtubules initially (B) and 2 min later (C). The enclosed region in panel A was taken from the authors' video $2 \mathrm{~A}$, whereas videos $\mathrm{B}$ and $\mathrm{C}$ were obtained from the authors' video $2 \mathrm{~B}$ (http://www.jcb.org/cgi/content/full/jcb. 200203150/DC1/2). Reproduced from The Journal of Cell Biology, 2002, Vol. 159, pp. 441-452 by permission of the Rockefeller University Press.

designated by the white arrow and the more haphazard movement of the one marked by the yellow arrow. Moreover, the "white" particle seems to move more rapidly and over a greater linear distance than the yellow one. Careful consideration of the video suggests the situation is more complex, however, because 1) all particles - red and greenare in motion; 2) some red particles seem to move in association with microtubules, albeit more slowly; 3 ) the microtubular framework, itself, is in motion; and 4) sometimes, the white particle seems to move in a directional manner independent of the blue framework. Observant students who examine the seven frames of the movie that were elided from the middle of the 14-min sequence reproduced in the authors' figure $4 \mathrm{~B}$ will also notice that the particle designated by the white arrow disappears and reappears, and they may wonder whether different viruses are being tracked by the white arrow.

The 15 images in the video were captured at 1-min intervals over $14 \mathrm{~min}$, and the movie is projected at a rate of 1 frame per second (fps). Clearly, the time-lapse nature of the video makes rapid features of particle behavior difficult to appreciate, and useful information was likely lost through

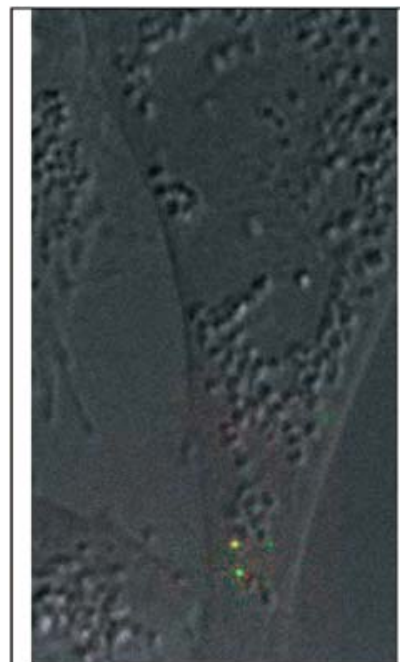

A

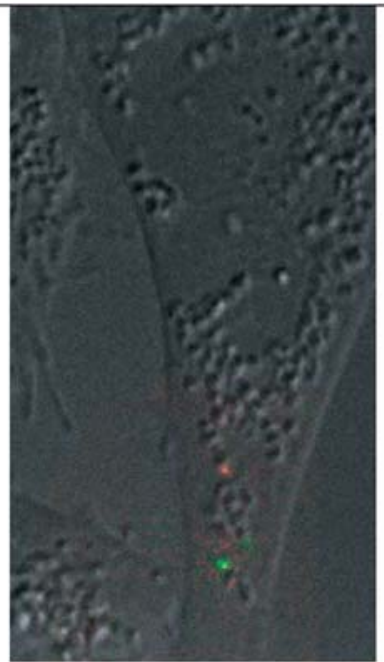

B

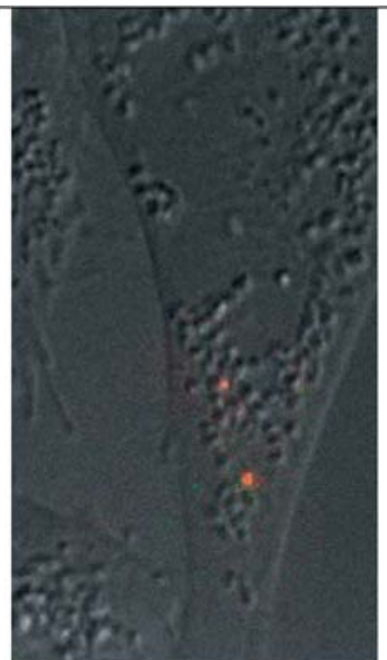

C

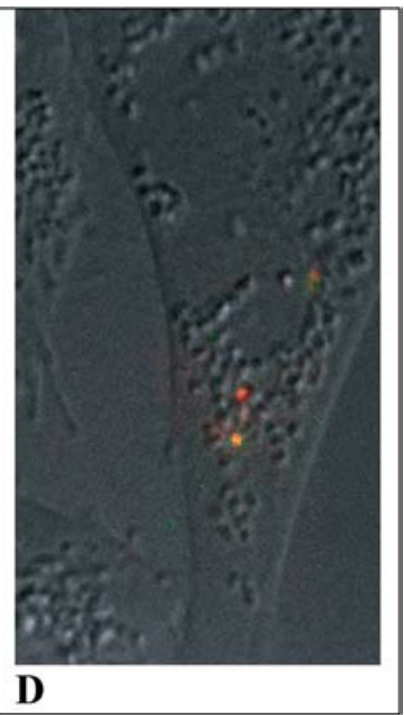

D

Figure 2. Fluorescence microscopic images showing the transport and acidification of two viral particles as they pass along the endosomal pathway, from early endosomes (A) toward the perinuclear region, after the passage of approximately 150 (B), 285 (C), and $345 \mathrm{~s}$ (D). Particle color reflects a standardized fluorescence ratio of $\mathrm{pH}$-sensitive and -insensitive dyes, ranging from green ( $\mathrm{pH} 8$ ) to red ( $\mathrm{pH} \leq 6)$. For orientation purposes, the fluorescent images of viral trajectories are projected over a single DIC image of the $\mathrm{CHO}$ host cell. The stills $(\mathrm{A}, \mathrm{B}, \mathrm{C}$, and D) were taken from the authors' supporting movie 2 (http://www.pnas.org/content/vol0/issue2003/images/data/0832269100/DC1/2269Movie2.mov). Copyright (2003) National Academy of Sciences, U.S.A. 


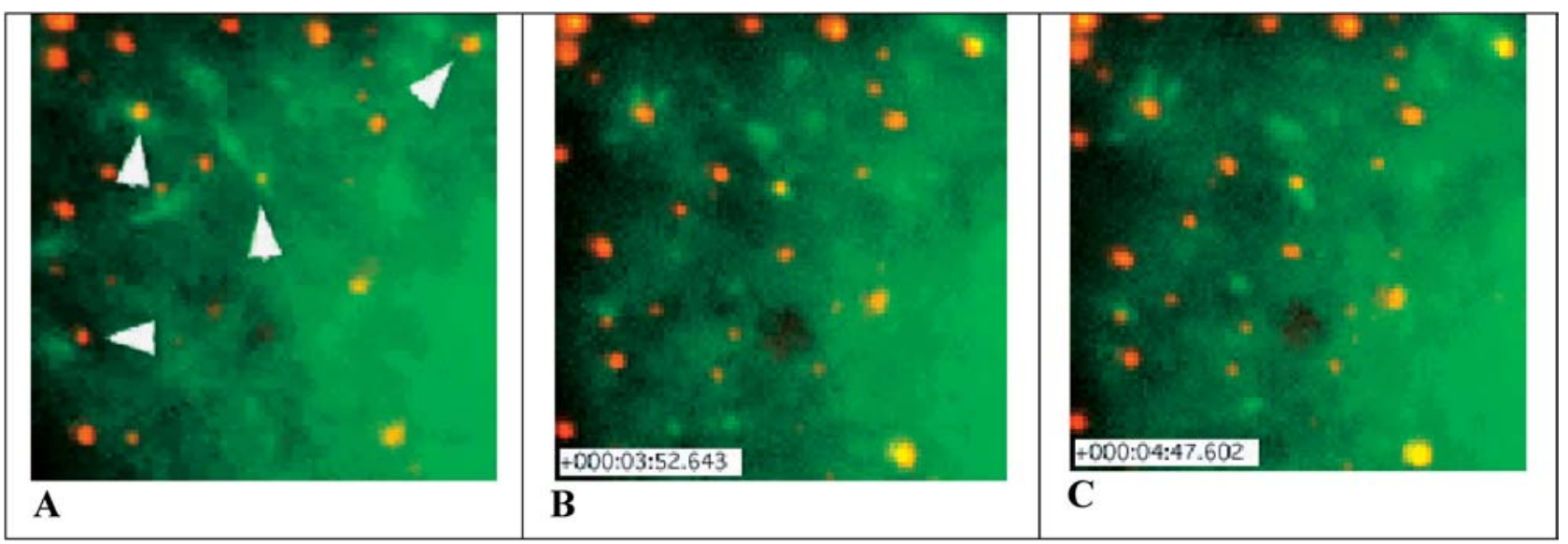

Figure 3. Fluorescence microscopic images of SV40 particles labeled with Alexa Fluor 594 (red) and associated with polymerizing GFP- $\beta$ actin (green). Induction of actin "tails" in association with labeled SV40 particles is indicated by the arrowheads in the center and upper left (A). Approximately $232 \mathrm{~s}$ have elapsed between panels A and B, and 281 s between panels A and C. The stills (A, B, and C) were obtained from the authors' movie 7A (http://www.sciencemag.org/content/vol296/issue5567/images/data/535/DC1/1069784S7A.mov). Reproduced by permission of the authors.

the time-lapse process. Students examining the video would benefit from a prior discussion of Brownian movement (and random walk behavior) and cytoskeleton-dependent directional movement (see Lodish et al., 2003). Most students would also more readily appreciate the types of particle movement if they mapped the particle paths in a manner similar to the authors' figure 3, with a transparent overlay on the QuickTime screen or video projection. Often, in an attempt to describe phenomena as clearly as possible, observers force observations into "either/or" dichotomous categories. Such oversimplification occurs, for example, when we distinguish Brownian from directional movement. When students track the intracellular paths taken by HIV, they will appreciate that individual particles exhibit both types of motion at different times. Further analysis of the directional movement also might lead to the hypothesis that HIV can move in association with cytoskeletal elements other than microtubules, and students should be encouraged to consider what those other elements might be and how they might be identified. The authors' data could then be consulted more closely for relevant experimental results. While outside the realm of the current study, students might also be interested in considering the motility of red particles (HIV still enclosed in VM): what sorts of movement are exhibited by these particles; where are they located; if still on the PM, how are they attached to the host cell; and how might such attachment sites move? Furthermore, how might all these questions be answered?

Students interested in other aspects of HIV might want to examine the authors' data concerning the location and function of reverse transcriptase within the green HIV particles and the ultrastructure of the reverse transcriptase particles.

\section{THE ENDOSOMAL PATHWAY OF INFLUENZA}

Given the cytoplasmic path taken by HIV, it would be interesting to know whether a similar path is taken by other infectious animal viruses. In this regard, a recent study of the flu virus would prove instructive (Lakadamyali et al., 2003; see also Alberts et al., 2002).
Like HIV, the influenza virus contains an RNA genome, a protein coat, and a VM. Unlike HIV, the flu virus replicates directly without a DNA provirus intermediate, and in the initial stage of infection, its VM does not fuse with the host cell PM. Rather, the virus is taken up by host cell endocytosis, and cytoplasmic entry of the viral core occurs when the VM fuses with the endosomal membrane in a $\mathrm{pH}$ sensitive manner (Smith and Helenius, 2004; Wagner and Hewlett, 1999). To characterize the invasion process, Lakadamyali et al. (2003) used fluorescence microscopy to follow virus labeled with lipophilic fluorescent dyes through endocytosis by Chinese hamster ovary $(\mathrm{CHO})$ cells. In one set of studies, the particles were doubly labeled with dyes whose fluorescence was sensitive (CypHer5) or insensitive (Cy3) to the $\mathrm{pH}$ of their environs. Following infection, fluorescence images were collected at the emission optima of each dye over a period of $450 \mathrm{~s}$ and at a rate of $2 \mathrm{fps}$. As the ratio of the emission of CypHer5 to $\mathrm{Cy} 3$ increased with a drop in $\mathrm{pH}$, fluorescence changed from green $(\mathrm{pH} 8)$ to yellow ( $\mathrm{pH} 7)$ and red $(\mathrm{pH}<6)$. For reference purposes, the sequential images of viral motility were superimposed on a single differential interference contrast (DIC) image of the host cell to create the authors' movie 2. Four frames from this movie are presented in Figure 2.

Watching the video, most students can immediately appreciate the drop in $\mathrm{pH}$ as endosomes containing labeled viruses move from the cell periphery to the perinuclear area and change their color from green to red. They also might appreciate the importance of a flu virus being uncoated in the vicinity of the host cell's endoplasmic reticulum. For comparison purposes, they will find it worthwhile to track the trajectories of the flu particles over time, as they did the HIV particles investigated by McDonald et al. (2003). Useful discussion can then be encouraged comparing the rates and paths of movement of "nude" HIV particles and endosomebound, intact flu virions. Distinguishing between random walk and directional movement might lead some students to distinguish three phases of flu infection (see the authors' figure 2), and the more inquisitive will likely want to know whether the directional movements of Phase II are actin or 
tubulin based. The authors test these alternatives using cytochalasin-D (a microfilament destabilizing drug), nocodazole (a microtubule destabilizing drug), and an antidynein antibody, and students should be encouraged to match the mechanism with predicted results before they consult the paper and the authors' figure 3. Inquisitive students might also want to see the results of treatment with an anti-kinesin antibody. Again, these test results can be compared with those obtained in the HIV study to develop a more general model of viral infection.

Many students who have worked their way through both papers and gained some understanding of the pathways followed by HIV and influenza might question the sweeping generality expressed in the opening sentence of the Abstract: "Influenza is a paradigm for understanding viral infection" (Lakadamyali et al., 2003). Comparative studies such as this provide an excellent opportunity for examining the nature of a scientific "paradigm," and students should be encouraged to evaluate the accuracy of the authors' claim (see also the review in the next section below). Possibly, discussion of this point could be extended fruitfully by students rewriting the opening statement in the Abstract in their own words (producing a "two-minute paper"; see Allen and Tanner, 2002). If they were later to refine their spontaneous revisions, they would find the excellent review by Smith and Helenius (2004) and more general comments by Alberts et al. (2002) especially useful.

\section{ROLE OF CAVEOLAE AND THE CYTOSKELETON IN INFECTION BY A NONENVELOPED VIRUS}

Students searching for a more rigorous paradigm for animal virus infection will find the study by Pelkmans et al. (2002) concerning the entry pathway of Simian virus 40 (SV40) particularly instructive. Lacking a VM envelope, SV40 invades host cells via invaginations of the PM called caveolae (see Watters, 2004). The viruses do not enter the endosomal pathway; rather, they are transported by vesicles to the endoplasmic reticulum (ER). It is unclear how SV40 enters or leaves the ER, but once in the cytoplasm, it is thought to enter the nucleus through nuclear pores (Pelkmans et al., 2001).

In support of their study, the authors provided an especially rich archive of 14 videos that documents various aspects of the invasion of SV40 into cultured African green monkey kidney cells (CV-1). SV 40 was labeled covalently with Alexa Fluor 594 (SV40-AF), which fluoresces red, whereas in different studies, components of caveolae and microfilaments, respectively caveolin (a coat protein) and $\beta$ actin, were labeled with GFP. The behavior of these components during viral infection was followed by fluorescence microscopy, and videos of cells containing a single fluorochrome are monochromatic; those involving two contrasting fluorochromes appear in color.

The videos in this series are quite interesting, but the images are complex and their meaning is not intuitively obvious; moreover, the article itself is densely detailed but sparse in methodology. Tackling the study is worthwhile, however, and I would consider introducing students to these videos in an indirect and selective manner. First, I would briefly describe SV40 and its entry pathway via caveolae, and then based on prior discussion of HIV and flu infectivity, I would ask them to postulate mechanisms for SV40 entry and transport by asking a series of focused questions: What do we know about caveolae? How do caveolae form? How could we prove SV40 entered via caveolae (and not through endosomes)? What sort of cytoskeletal transport system might be responsible for the transport of "caveosomes" (SV40 in caveolae-derived vesicles) to the ER? Then we would explore the videos and the article as we tried to answer these questions. For example, the authors' movie 5 depicts the association of SV40-AF with caveolin-GFP at the host cell surface (presumably coating the PM invaginations), and the authors' movie 7A depicts the association of polymerizing GFP-actin with SV40-AF (presumably in vesicles derived from caveolae), suggesting that microfilament assembly plays a role in viral movement through the cytoplasm. Three stills from movie 7A are presented in Figure 3, which illustrate the association of polymerizing actin "tails" with SV40 vesicles.

Many students might want to test the involvement of microfilaments in SV40 transport, leading to a discussion of the inhibitory effects of Latrunculin A (LatA, which binds with monomeric actin and prevents its polymerization) and to an examination of the authors' movie 9. The actin cytoskeleton might play another, earlier role during infection, as suggested by the authors' movie 5A, which depicts the effects of LatA on the movement of virus-loaded GFPcaveolae. In this respect, more advanced students might want to explore the possible involvement of dynamin (a protein activator of endocytosis) and of various protein kinases in SV40-induced caveolae formation, and the article and various movies provide interesting data for their consideration.

Students who have come to these videos and SV40 infectivity from prior studies of other viral-host cell systems might want to know whether caveosomes also move along the microtubule transport system. This question is not addressed by Pelkmans et al. (2002), but a later review from the same laboratory provides more comparative details about viral movement along the various cytoskeletal elements (Smith and Helenius, 2004).

\section{BROWSING FOR RESEARCH VIDEOS: AN ASIDE}

Readers interested in browsing online for interesting research videos will find that some journal archives and search engines are easier to use than others. As should be evident from the preceding discussions and from a past column, I consider the entry provided by The Journal of Cell Biology and its Annotated Video Collection, the most userfriendly I have encountered so far. Other journals have yet to provide readers with such a welcoming portal, and finding videos in their archives requires judicial use of search engines and carefully chosen key words or, in the extreme, tedious browsing of various tables of contents. Even when archives identify which articles contain supplemental material, terminology changes, sometimes without notification, and although similar, different key words and phrases must be used for finding video material. For example, although rich with video records, browsers of the PNAS archives will find such material catalogued in archives of earlier issues as "supplemental movies" and then later as "supporting movies." Thus, using the Advanced Search Mode at www.pnas.org, a Text/Author/Title field entry of "supporting movie" produced a list of 149 articles with video records 
from July 2001 through early July 2004. Performing the same search for the combination of "supplemental movie" and "supporting movie" produced 177 records from early March 1999 through early July 2004. A more basic search with simply "movie" produced 283 hits, but many of these articles, and all prior to 1999, lacked video records (and presumably referred the reader to articles containing data derived from such movies). As an additional benefit, the PNAS bibliographies can be further refined with disciplinary categories assigned to the articles it publishes, which appear in a small menu box in the upper right of the search screen. Most of the videos in the list of 177 records deal with biological phenomena.

Unfortunately, without a specific citation, finding videos in Science (www.sciencemag.org) is more difficult, and successful foraging requires several trials with different key words to produce useful results. For example, a search in the Keyword(s) Field for "movie" produced 170 video-containing articles (between July 1999 and July 2004), but many of these, especially among the earlier records, lacked video supplements. With the more lengthy Boolean string "("Supporting Online Material" or "Supplemental Data") and movie and cell" produced a more useful list of 77 citations for the same period: many of these contained video records. Several very interesting videos in this list depict physical phenomena such as crystal growth and surface adsorption, although most deal with biological phenomena.

Incidentally, while browsing the Science archives, I unexpectedly discovered an interesting review (Sibley, 2004) concerning invasive mechanisms employed by the Apicomplexa parasites (including the malaria-causing Plasmodium). Last year in this column, I reviewed two sets of videos from the Sibley Lab describing the motility of these parasites (Watters, 2003), and readers wanting more information about the locomotion of these cells might be interested in the Science article and its 10 appended movies. Produced by three different laboratories and depicting the motility of Plasmodium, Toxiplasma, and two related species, these more recent videos significantly extend our under- standing of how these parasites move and invade their host cells.

\section{REFERENCES}

Alberts, B., Johnson, A., Lewis, J., Raff, M., Roberts, K., and Walter, P. (2002). Molecular Biology of the Cell, 4th ed. New York: Garland Science.

Allen, D., and Tanner, K. (2002). Approaches in cell biology teaching. Cell Biol. Educ. 1(1), 3-5.

Goldsby, R.A., Kindt, T.J., Osborne, B.A., and Kuby, J. (2003). Immunology, 5th ed. New York: W.H. Freeman.

Lakadamyali, M., Rust, M.J., Babcock, H.P., and Zhuang, X. (2003). Visualizing infection of individual influenza viruses. Proc. Natl. Acad. Sci. USA 100(16), 9280-9285.

Lodish, H., Berk, A., Matsudaira, P., Kaiser, C.A., Krieger, M., Scott, M.P., Zipursky, S.L., and Darnell, J. (2003). Molecular Cell Biology, 5th ed. New York: W.H. Freeman.

McDonald, D., Vodicka, M.A., Lucero, G., Svitkina, T.M., Borisy, G.G., Emerman, M., and Hope, T.J. (2002). Visualization of the intracellular behavior of HIV in living cells. J. Cell Biol. 159(3), 441452.

Pelkmans, L., Kartenbeck, H., and Helenius, A. (2001). Caveolar endocytosis of simian virus 40 reveals a new two-step vesiculartransport pathway to the ER. Nature Cell Biol. 3, 473-483.

Pelkmans, L., Puntener, D., and Helenius, A. (2002). Local actin polymerization and dynamin recruitment in SV40-induced internalization of caveolae. Science 296(5567), 535-539.

Sibley, L.D. (2004). Intracellular parasite invasion strategies. Science 304(5668), 248-253.

Smith, A.E., and Helenius, A. (2004). How viruses enter animal cells. Science 304, 237-242.

Wagner, E.K., and Hewlett, M.J. (1999). Basic Virology. Oxford: Blackwell Science.

Watters, C. (2003). Video views and reviews. Cell Biol. Educ. 2, 210213.

Watters, C. (2004). Video views and reviews: Golgi export, targeting and plasma membrane caveolae. Cell Biol. Educ. 3, 52-56. 